\title{
Erratum zu: DUBBEL, 25. Aufl. ISBN 978-3-662-54804-2
}

K.-H. Grote, Magdeburg; B. Bender, Bochum; D. Göhlich, Berlin

Die Reihenfolge der Autoren in den Kapiteln G1, G1.1, G1.2 und G1.3 war nicht korrekt und wurde wie folgt geändert:

G1 Bauteilverbindungen

H. Wohlfahrt†; T. Widder, Braunschweig; M. Kaßner†;

K. Thomas †; K. Dilger, Braunschweig; H. Mertens, Berlin;

R. Liebich, Berlin

\section{G1.1 Schweißen}

K. Dilger

Kapitel basiert auf: H. Wohlfahrt $\dagger$, K. Thomas $\dagger$, M. Kaßner $\dagger$

\section{G1.2 Löten und alternative Fügeverfahren}

K. Dilger

Kapitel basiert auf: H. Wohlfahrt $\dagger$, K. Thomas $\dagger$, M. Kaßner $\dagger$

\section{G1.3 Kleben}

K. Dilger

Kapitel basiert auf: T. Widder

Ebenso wurde die Autorenreihenfolge der Kapitel O1, O2 und $\mathrm{O} 3$ berichtigt:

\section{O1 Schwingungen}

H. Hanselka, Karlsruhe; S. Herold, Groß-Umstadt; R. Nordmann, Darmstadt; T. Nestorović, Bochum

Überarbeitet durch T. Nestorović

O2 Kurbeltrieb, Massenkräfte und -momente, Schwungradberechnung

R. Nordmann, Darmstadt; T. Nestorović, Bochum

Überarbeitet durch T. Nestorović

O3 Maschinenakustik

H. Hanselka, Karlsruhe; J. Bös, Darmstadt; T. Nestorović, Bochum

Überarbeitet durch T. Nestorović 